\title{
Pericardial Tamponade: Diagnostic and Evolutive Aspects in a Cardiology Department in Dakar, Senegal
}

\author{
Bodian M11, Aw F1, Mingou JS1*, Ndao JS1, Sarr SA1, Ngaïdé AA², \\ Dioum $M^{3}$, Beye $\mathrm{SM}^{5}$, Ndiaye $\mathrm{MB}^{1}$, Archich N1, Sangaré $\mathrm{Z}^{1}$, Mbaye $\mathrm{A}^{2}$, \\ Kane $\mathrm{Ad}^{5}$, Diao $\mathrm{M}^{1}$, Kane $\mathrm{A}^{4}$ and $\mathrm{Ba} \mathrm{SA}^{1}$ \\ ${ }^{1}$ Department of Cardiology, Aristide Le Dantec Hospital University, Dakar, Senegal \\ 2Department of Cardiology, HOGGY, Dakar, Senegal \\ ${ }^{3}$ Department of Cardiology, Fann Hospital University, Dakar, Senegal \\ ${ }^{4}$ Departement of Cardiology, DalalJamm Hospital, Dakar, Senegal \\ 5Departement of Cardiology, Regional Hospital of Saint Louis, Senegal
}

\begin{tabular}{|c|}
\hline Research Article \\
Volume 1 Issue 2 \\
Received Date: November 14, 2017 \\
Published Date: November 27, 2017 \\
\hline
\end{tabular}

*Corresponding author: Joseph Mingou, Cardiology Department, Aristide Le Dantec Hospital University, Dakar, Senegal, Email: mingoujoseph@gmail.com

\begin{abstract}
Tamponade is one of the major complications of pericarditis which is immediately life threatening. The objective of the study was to evaluate the diagnostic and evolutive aspects of pericardial tamponade in a cardiology department of Dakar. We included 40 patients who had a tamponade with a file that could be exploited over a period of 3 years and 6 months (January 2010 to June 2014). The mean age of the patients was 41 years (range between 15 and 80 years). There was a male predominance with a sex ratio (23/17) of 1.35 .

Signs were dominated by dyspnea (95.0\%), pain (85.0\%) and fever (40\%). Signs of poor hemo dynamic tolerance were evidenced by weak peripheral pulses (21.16\%), paradoxical pulse (5\%), and hypotension and/or shock (15.0\%).

The diagnostic means, besides the clinic examination, was mainly by conducting an echocardiography. Thus, there was a proto-mesodiastolic collapse of RV in $82.5 \%$ of cases, a telediastolic collapse of the right atrium in $87.5 \%$ of cases, a fibrin network in $55 \%$ of cases.

Emergency treatment with per cutaneous pericardiocentesis was performed in all our patients using the subxiphoid approach. There was a favorable evolution in $77.5 \%$ of cases. Were corded 8 cases (or $20 \%$ ) of death in all admitted cases. Pericardial tamponade is a medical emergency. The diagnosis is easy using echocardiography but the etiological diagnosis is always problematic in our working context.
\end{abstract}

Keywords: pericarditis; Tamponade; pericardial effusion; pericardial puncture; Dakar 


\section{Open Access Journal of Cardiology}

\section{Introduction}

Tamponade is one of the major complications of pericarditis which is immediately life threatening. This is due to an acute or sub acute compression of the heart by a pericardial effusion of rapid accumulation or large amount of fluid. From a clinical viewpoint, the hemodynamic profile of pericardial tamponade requires in practice only a reduced para clinical assessment, often limited to conducting an echocardiography. In Africa, the most common etiology is tuberculosis, which has been rising sharply since the advent of HIV infection [1]. The difficulties associated with delayed diagnosis and treatments have a negative impact on the prognosis of this complication. This study was carried out with the aim of evaluating the diagnostic and therapeutic aspects of pericardial tamponade in our setting.

\section{Patients and methods}

This is a descriptive retrospective study carried out at the cardiology department of the Aristide Le Dantec University Hospital in Dakar over a period of 3 years and 6 months (January 2010 to June 2014).

We included all patients who were admitted for pericardial tamponade. All patients have undergone a clinical examination and had para clinical tests including an echocardiography.

The parameters studied in this study were: epidemiological aspects, clinical signs (complete clinical examination) and para clinics (laboratory, electrocardiogram, X-rays, echocardiography), and therapeutic aspects (Figure 1).

A questionnaire was used to collect required information from patients' clinical records. These data have been entered and evaluated using the EPI INFO 7 software.

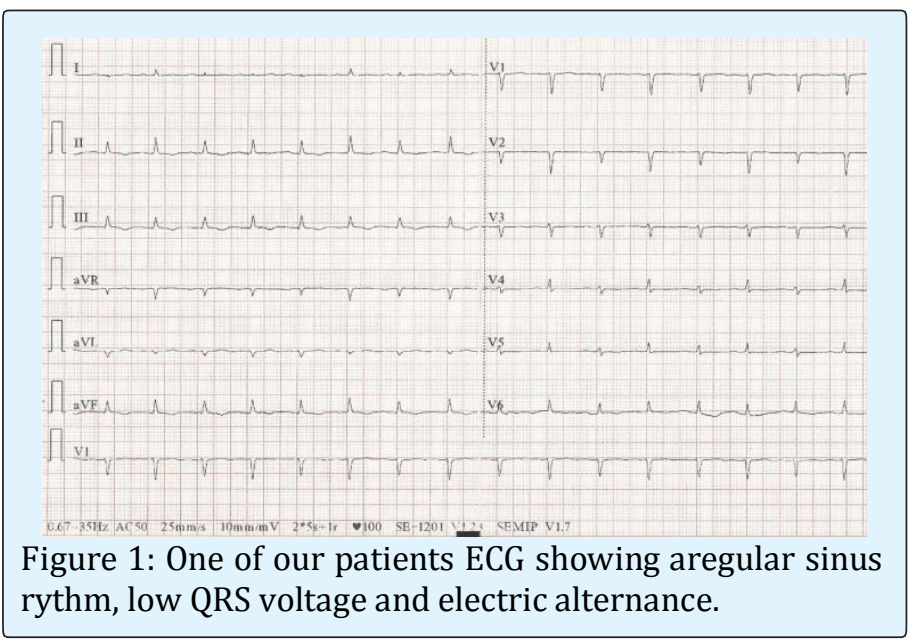

\section{Results}

Records of forty patients admitted for pericardial tamponade over the study period were reviewed. The mean age of patients was 41 years with extremes of 15 and 80 years. The age group 21-40 was more represented with $42.5 \%$ followed by the $41-60$ age groups. There was a male predominance with a sex ratio (23/17) of 1.35 . Married patients accounted for $65.5 \%$ of the population. Patients had a history of pulmonary tuberculosis, HIV seropositivity, and alcohol/tobacco consumption in 5\%, respectively. Hypertension was found in $12.5 \%$ of individuals. The majority of patients (71\%) were of low socioeconomic status.

Presenting symptoms were dyspnea (95.0\%), pain $(85.0 \%)$ and fever $(40 \%)$. Pain was pricking in $45 \%$ of cases, burning, constrictive and heavy in $15.0 \%$ of cases each respectively, and twisting sensation in $10 \%$ of patients. Physical examination noted a poor general condition (32.5\%), a muffling of the heart sounds $(67.5 \%)$, weak pulses $(30.0 \%)$, increased venous pressure $(75.0 \%)$, a paradoxical pulse $(5 \%)$, hypotension and / or shock (15.0\%).

The tuberculin skin (Mantoux) test was positive in 64\% of the cases.

Laboratory assessment found anemia in $85.8 \%$ of individuals with an average hemoglobin level of $9.8 \pm 2.4$ ( 2 and $16 \mathrm{~g} / \mathrm{dl}$ ). The anemia was hypo chromic microcytic in $95.0 \%$. An inflammatory syndrome was noted in $92.9 \%$ of cases.

Bacteriological examination of the pericardial fluid was positive in 2 cases, isolating staphylococcus spp and streptococcus pneumonia in one case each. The examination of AFB sputum was negative in all patients who had done so.

The ECG had a tachycardia in $90 \%$ of cases, a Holzmann stage (I to III) in $51.8 \%$ of cases, electrical alternation in $15 \%$ of patients and micro voltage in $100 \%$ of cases. A supra ventricular rhythm disorder was noted in $20 \%$ of patients, $17.5 \%$ of whom by fibrillation.

All our patients had cardiomegaly with an average cardio-thoracic ratio at 0.65 (0.6 and 0.8$)$. The cardiophrenic angle was open in $70 \%$ of the chest $x$-rays.

Trans-thoracic echocardiography (TTE) recorded a circumferential effusion in all patients with an average thickness of $33 \mathrm{~mm}$ (16 and $56 \mathrm{~mm}$ ) in the short- axis view. The effusion was of large abundance in $86 \%$ of cases. A proto-meso diastolic collapse of right ventricle 


\section{Open Access Journal of Cardiology}

right atrium and a swinging heart pattern were noted in $82.5 \%, 87.5 \%$ and $22.5 \%$ respectively. A fibrin network was visualized in $55 \%$ of individuals.

The etiologies were represented by tuberculosis in 17 cases, bacterial (pyogenic) infection in 10 purulent cases, HIV in 5 patients and uremic renal insufficiency in 1 case. In 7 patients we could not establish an etiological diagnosis.

Emergency treatment with per cutaneous pericardiocentesis was performed in all our patients. It was performed exclusively by the subxiphoid approach and was non-ultrasound guided. The procedure was successful in a single attempt in $55.6 \%$ of the cases.

The average fluid quantity obtained was $1022 \mathrm{ml}$ with extremes of 50 and $3100 \mathrm{ml}$. The puncture fluid was serohematic in $55 \%$ of cases, yellowish in $25 \%$ of cases and purulent in $20 \%$ of cases. A fibrin network was noted in $55 \%$ of cases.

Non steroidal anti-inflammatory drugs were used in $77.5 \%$ of cases; anti tuberculosis in $42.5 \%$ of cases, corticosteroids in $37.5 \%$ and nonspecific antibiotics in $25 \%$ of patients. Colchicine was administered to a patient $(2.5 \%)$.

The immediate evolution was favorable in $77.5 \%$ of the cases. We recorded 8 cases (or 20\%) of death in all admitted cases. Two cases of recurrence of tamponade were noted. At six months, there were 5 other deaths (sixmonth mortality of $32.5 \%$ ), 4 chronic pericarditis and 10 patients lost to follow-up.

\section{Discussion}

The incidence of tamponade is $34.18 \%$ of patients hospitalized for pericardial effusion in our cardiology department [2]. The estimated incidence in Marseille was between 20 and $50 \%$ in patients with autoimmune disease, 30\% in hypothyroid patients and 35\% in patients with cancer (lung, breast and lymphoma). The average age of our patients, 41 years, is almost identical to that described in sub-Saharan Africa $[1,3,4]$. In addition, the age group 21 - 40 was the most representative in our study at $42.5 \%$. This is partly due to the fact that tuberculosis is the main aetiology that has gained renewed interest with the advent of HIV/AIDS, commonly found in young adults. A male predominance was noted with a sex ratio $(\mathrm{M} / \mathrm{F})$ at 1.35 .

Signs were dominated by dyspnea $[5,6]$. According to Kerbaul F., the clinical picture consists of dyspnea of aggravating stress, associated with a sensation of chest tightness and nonspecific tachycardia. At the extreme, the patient is orthopneic and refuses the dorsal decubitus position [7]. For example, dyspnea, chest pain and tachycardia were $95.0 \%, 85.0 \%$ and $72.5 \%$, respectively. Because of the inability to increase the systolic ejection volume, the heart is forced to accelerate its frequency to maintain sufficient cardiac output, this is the threshold of de compensation. Exceeding this limit, the blood flow falls. Thus, $15.0 \%$ of our patients had hypotension and / or shock. Increased venous pressure was noted in $75.0 \%$ of our patients, compared with $22 \%$ for Kerbaul in France [7] and a muffling of heart sounds in $67.5 \%$ vs $52 \%$ for Sidiki in Guinea (Figure 2).

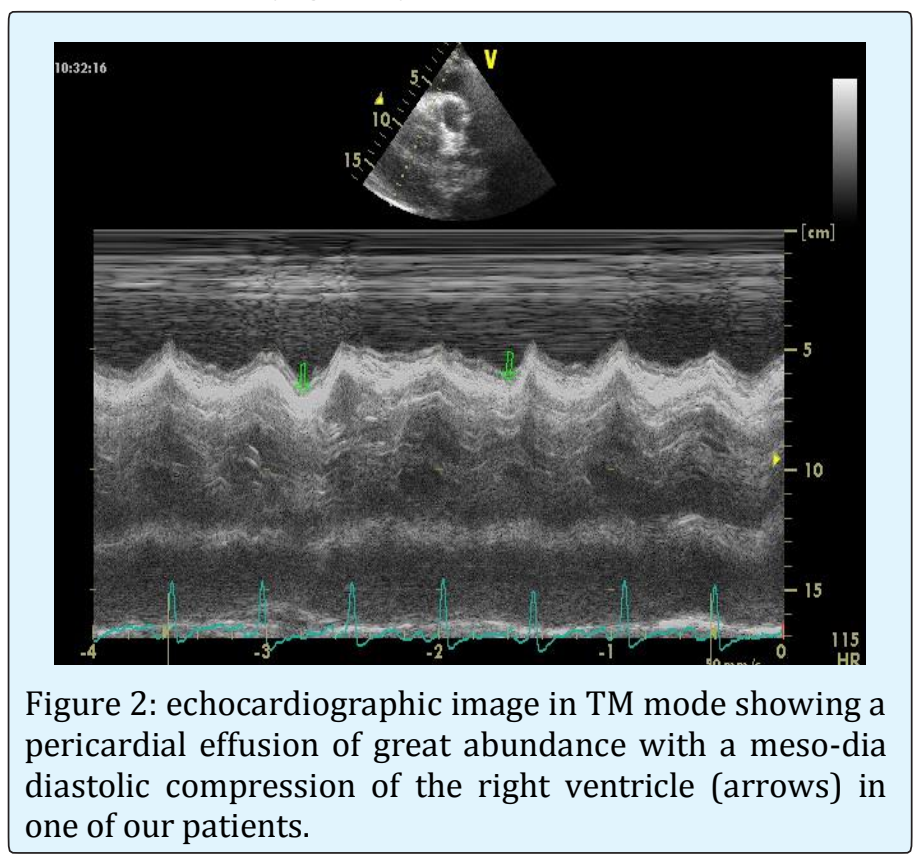

The biological inflammatory syndrome has only an orientation value. The bacteriological examination of pericardial fluid is most often negative in our regions (85\%) and the search for non-contributing acid-fast bacilli.

The ECG is sometimes useful for diagnosis especially in our regions where the technical capacity is limited. It attracts our attention to peripheral and / or diffuse micro voltage, a non-specific sign, but of high orientation value. All our patients had this sign. In addition, the ECG may indicate signs of poor tolerance including electrical alternation which was noted in $15.0 \%$ of our patients.

Echocardiography is an essential examination in the diagnosis and etiologic orientation of tamponade. Indeed, the positive diagnosis of tamponade by TTE is easy. However, its etiology remains an enigma in our regions and the TTE is a great contribution in this approach by appreciating, besides the abundance, the appearance of 


\section{Open Access Journal of Cardiology}

the liquid, the presence or not of fibrin. Tuberculosis is the most common etiology in our country. It is characterized by an abundance of pericardial effusion, often circumferential, which can rapidly involve the vital prognosis by a tamponade, the presence of fibrin network, a sero-hematic fluid predominantly lymphocytic $[8,9]$. In our study, echocardiography noted the presence of a fibrin network in $55.0 \%$ of individuals and the puncture fluid was sero-hematic in the same proportions. In addition to diagnosis, the TTE allows the indication and control of pericardiocentesis, the only life saving procedure in this type of emergency (class I recommendation) [2] (Figure 3).

Removal of fluid, even in small quantities (30 - $50 \mathrm{ml}$ ), makes it possible to stop the compression and relieve the patient.

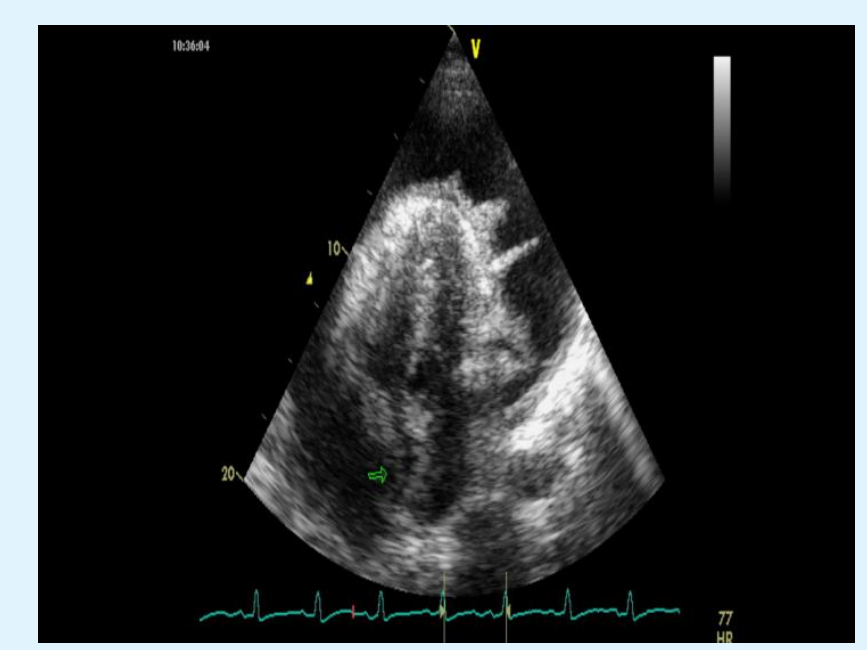

Figure 3: Echo cardio graphic image in 2D showing a pericardial effusion of great abundance with compression of the right atrium (arrow) and the presence of fibrin network in one of our patients.

The prognosis of this complication is intimately linked to its cause and therefore to its proper management by a specific treatment. Thus, patients received antituberculosis drugs in $37.5 \%$, antibiotics in $25.0 \%$. To avoid evolution towards constriction in bacterial infections specific or not, corticosteroid therapy is necessary in association with the specific treatment. The use of corticosteroids is controversial. However, studies in the literature $[10,11,12]$ suggest that their association with specific therapy is associated with decreased mortality, decreased frequency of pericardiocentesis or pericardiotomy. Non steroidal anti-inflammatory drugs were administered to $77.5 \%$ of patients according to guidelines [13] (Figure 4).

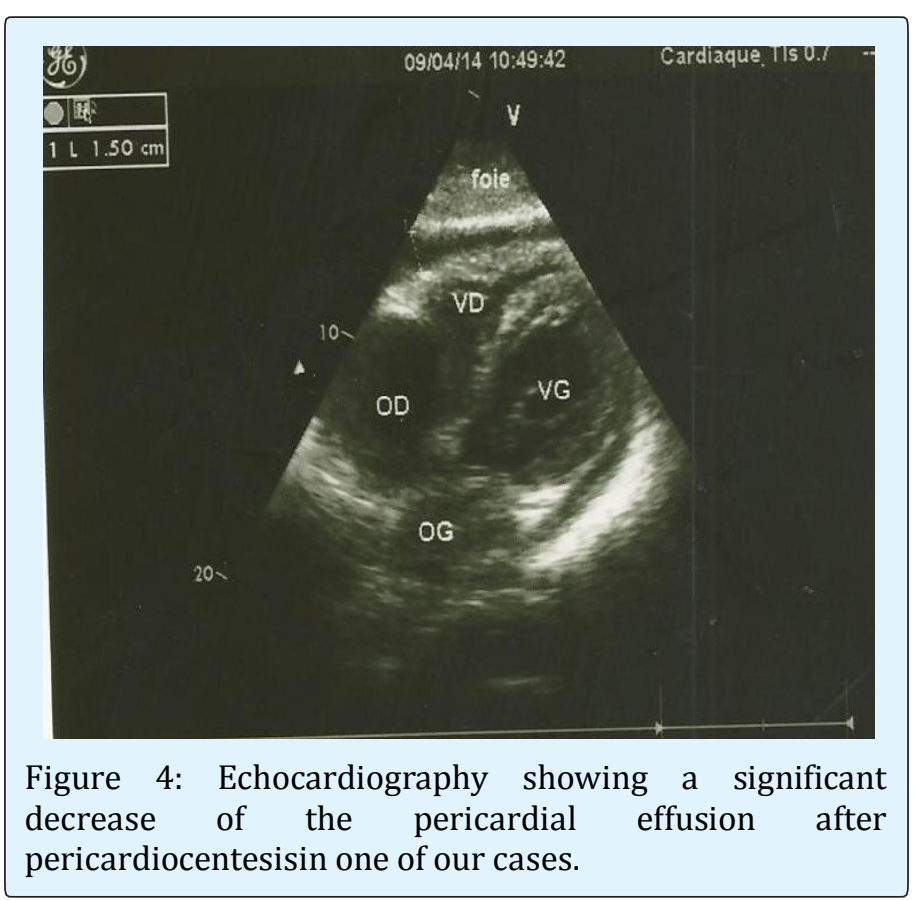

The evolution under treatment is a valuable argument in the management of this complication in our working context as in the African series [14]. Mortality was high, hospitalized at $20.0 \%$ and overall mortality at 6 months of $32.5 \%$. This is in practice related to delay in seeking healthcare, diagnostic problems, the underlying or predisposing factors, the cause, the therapeutic difficulties and the modest resources of the patients. During the follow-up 4 cases $(10 \%)$ of chronic pericarditis were noted. Indeed, chronic constrictive pericarditis must always be of concern. Imazio [15] reported in a study of a cohort of 500 cases of pericarditis a constriction rate of 31.65 per 1000 for tuberculous pericarditis. This rate could reach 52.74 per 1000 when they became purulent.

\section{Conclusion}

Pericardial tamponade is a frequent complication of pericarditis with effusion especially of tuberculous origin. If the diagnosis is easy through echocardiography, the etiological diagnosis is always problematic in our working context. Pericardiocentesis is the urgent treatment for this complication, but the actual management should be directed to that of the cause, using clinical, biological and ultrasound information. The high mortality of this complication calls for preventive measures such as pericardial drainage of high-abundance effusions, particular interest in tuberculosis screening in urban but especially rural areas, and early and adequate management of pericarditis. 


\section{Open Access Journal of Cardiology}

\section{Limitations of the Study}

The retrospective nature of the study could be a source of bias in our study. A lot of data could also have been lost.

\section{Conflicts of Interest}

The authors do not declare any conflict of interest.

\section{Contribution of Authors}

All authors contributed to this work. They read and approved the final version of the manuscript.

\section{References}

1. Yaméogo AA, Kyelem CG, Nikiéma Z, Birba E, Yaméogo TM, et al. (2012) Les péricardites tuberculeuses au centre hospitalier universitaire de Bobo-Dioulasso, Burkina Faso. Pan African Medical Journal 12(16): 1-5.

2. Maisch B, Seferović PM, Ristić AD, Erbe IR, Rienmüller $\mathrm{R}$, et al. (2004) Guidelines on the Diagnosis and Management of Pericardial Diseases Executive Summary: The Task Force on the Diagnosis and Management of Pericardial Diseases of the European Society of Cardiology. Eur Heart J 25 (7): 587-610.

3. Sidiki MA (2012) Péricardites : prise en charge et pronostic au service de chirurgie thoracique de l'hôpital National Donka. Etude de 19 cas, Thèse Med Guinée Bissau.

4. Damorou F, Soussou B, Adjenou K, Goeh-Akue E, Mawoussi A, et al. (2005) Tamponnade cardiaque non-traumatique: Intérêt de l'Echo doppler cardiaque et prise en charge. J Recher Sci Lomé 7 (1): 6-8.

5. Sagristà-Sauleda J, Angel J, Sambola A, Alguersuari J, Permanyer-Miralda G, et al. (2006) Low-pressure cardiac tamponade : clinical and hemodynamic profile. Circulation 114 (9): 945-952.
6. Roy CL, Minor MA, Brookhart A, Choudhry NK (2007) Does This Patient With a Pericardial Effusion Have Cardiac Tamponade ? JAMA 297(16): 1810-1818.

7. Kerbaul F, Michelet P (2013) Tamponnade cardiaque $1-14$

8. Fatimi SH, Faheem-ul-Haq, Jalil F, Muzaffar M, Hanif HM (2011) Tuberculous pericardial abscess with impending pericardial effusion and cardiac tamponade. J Pak Med Assoc 61(3): 286-287.

9. Mianfoutila S, Nkiwabonga L, Nkoua JL (1996) Drainage chirurgical du péricarde à propos de 10 cas. Médecine d'Afrique Noire 43: 8-9.

10. Mayosi BM, Ntsekhe M, Volmink JA, Commerford PJ (2002) Interventions for treating tuberculous pericarditis, Cochrane Database Syst Rev (4): CD000526.

11. Ntsekhe M, Wiysonge C, Volmink JA, Commerford PJ, Mayosi BM (2003) Adjuvant corticosteroids for tuberculous pericarditis: promising, but not proven. QJM 96: 593-599.

12. Evans DJ (2008) The use of adjunctive corticosteroids in the treatment of pericardial, pleural and meningeal tuberculosis: do they improve outcome? Respir Med 102(6): 793-800.

13. Adler $Y$, Charron $P$, Imazio $M$, Badano L, BarónEsquivias G, et al. (2015) 2015 ESC Guidelines for the diagnosis and management of pericardial diseasesWeb Addenda. European Heart Journal 36(42): 29212964.

14. Bouakez-Ajabi A, Bouakez H, Zaouali RM (1999) Les péricardites-Aspects cliniques et étiologiques. Médecine du Maghreb 78: 29-31.

15. Imazio M, Brucato A, Maestroni S, Cumetti D, Belli R, et al. (2011) Risk of constrictive pericarditis after acute pericarditis. Circulation 124(11): 1270-1275. 